Instituto Internacional de Investigación y Desarrollo Tecnológico Educativo INDTEC, C.A.

DOI: https://doi.org/10.29394/scientific.issn.2542-2987.2017.2.4.17.302-318

OAI-PMH: http://www.indteca.com/ojs/index.php/Revista Scientific/oai

\title{
Integración de los Saberes Comunitarios como Estrategia de Aprendizaje en la Praxis Educativa de la Comunidad Escolar los Llanitos de Timotes
}

\author{
Autora: Yajaira Esperanza Araujo Araujo \\ Universidad Nacional Experimental "Rafael María Baralt", UNERMB \\ araujoyajairaunica@hotmail.com \\ Trujillo, Venezuela
}

\section{Resumen}

La educación fortalece la identidad del individuo, generando transformaciones sociales que permiten mejorar la calidad de vida, por ello el presente artículo, consiste en divulgar algunos de los avances del trabajo de investigación que se está desarrollando en la comunidad escolar de los Llanitos de Timotes, el cual tiene como propósito general La Integración de los Saberes Comunitarios como estrategia de aprendizaje en la praxis educativa, se sustenta en la teoría crítica, bajo las directrices de la Ley Orgánica de Educación (2009), resolución 015 del Viceministerio de Comunidades Educativas y Unión con el Pueblo. Metodológicamente fue una Investigación Acción Participativa de naturaleza analítica, documental y reflexiva. Como resultados parciales se obtuvo que la escuela debe proyectar estrategias hacia la comunidad aprovechando sus saberes e incorporarlos al proceso educativo, para que los alumnos sean más conscientes de la realidad en que viven y valoren lo que los rodea. A medida de reflexión y miras a la transformación curricular se concluye que la escuela como centro de formación no solo tiene la función de transmitir conocimientos sino además proteger el acervo socio cultural, promover valores y saberes de identidad, integrando a la comunidad como fuente de sabiduría para enriquecer la praxis pedagógica.

Palabras claves: integración; saberes; cultura; valores. 


\title{
Integration of Knowledge Community Learning as a Strategy in Educational Praxis Community School Timotes los Llanitos
}

\begin{abstract}
Education strengthens the individual's identity, generating social transformations that improve the quality of life, so this article is to disclose some of the progress of the research work being developed in the school community Llanitos Timotes, which has the general purpose Integrating community knowledge as a learning strategy in educational practice, is based on critical theory, under the guidelines of the Organic Law of Education (2009), resolution 015 of the Vice Ministry of educational Communities and Union with the People. Methodologically was a Participatory Action Research analytical nature, documentary and reflective. As partial results was obtained that the school should project to the community strategies leveraging their knowledge and incorporate the educational process, so that students are more aware of the reality in which they live and appreciate their surroundings. As of reflection and a view to the curriculum transformation it is concluded that the school as a training center not only has the function of transmitting knowledge but also protect the socio-cultural heritage, promote values and knowledge of identity, integrating the community as a source of wisdom to enrich the pedagogical praxis.
\end{abstract}

Keywords: integration; knowledge; culture; values.

Date Received: 22-10-2016

Date Acceptance: 12-01-2017 


\section{Introducción}

En la actualidad educar quiere decir formar ciudadanos y ciudadanas, los cuales no están parceladas en compartimientos, ni en capacidades aisladas; la capacidad de una persona para relacionarse depende de las experiencias que vive, y la educación es un lugar preferente para establecer vínculos e interacciones que condicionan y dependen de las propias concepciones sobre uno mismo lo que da y sobre los demás. Entender esta transformación exige un modelo pedagógico en que la escuela se configura como un microsistema por la teoría-praxis, con una organización social y unas relaciones interactivas entre el vínculo hogar, escuela comunidad a los procesos pedagógicos y los incorpore como parte fundamental a la formación integral de él y los educandos.

Ante esta realidad, en aspectos relacionados con la educación, se orienta el horizonte escogido en lo concerniente a la calidad educativa. La UNESCO en la declaración de la educación para todos, plantea que la educación es tarea de todos porque supone compromisos renovados, de sus actores en espacios de corresponsabilidad. Nuestro país a raíz de la promulgación de la Constitución de la República Bolivariana de Venezuela (1999), la participación se asume como la columna vertebral de todos los proyectos sociales, porque proporciona oportunidades al ser humano para que ejerza su derecho como protagonista de su destino, al tiempo que busca la igualdad de oportunidades.

El presente artículo tiene como propósito divulgar algunos avances relacionados con el trabajo de investigación denominado La Integración de los Saberes Comunitarios como Estrategia de Aprendizaje en la Praxis Educativa de la Comunidad de los Llanitos de Timotes; para que la escuela desde la dimensión socioeducativa emprenda la integración activa con la idea de reivindicar el sujeto como constructor de su propia comunidad y de la calidad educativa, y a su vez la forma más rica de los valores culturales y la 
conservación de conocimientos históricos, religiosos o de confrontación de saberes sociales para resguardar la identidad etnográfica del sector.

\section{Sustentación epistemológica}

Matos, Y. (2012) señala que, para la construcción de conocimientos en colectivo durante la investigación, se requiere de un investigador social con ética, con conocimientos y valores que contribuyan a un interactuar desde el diálogo intersubjetivo, así como de un compartir de saberes. En tal sentido, se requiere que como investigador se actúe como un participante más de ese proceso transformador en y desde la comunidad.

Visto de esta manera, se debe indagar el mundo histórico social de las personas tomando como vía la extracción de la información a partir del método fenomenológico hermenéutico. En este sentido, el presente trabajo se desarrolla desde el paradigma socio crítico reflexivo, donde se asume ontológicamente, que la realidad no es aprehensible como un hecho objetivo; por el contrario, la misma es un proceso de construcción individual y colectiva, donde los elementos y dimensiones inherentes a los sujetos que participan en el fenómeno estudiado, como la posición del propio investigador, aporte elementos al proceso mismo de construcción del conocimiento.

En el componente epistemológico, se reconoce la relación sujetoobjeto, como un vínculo inquebrantable. El análisis de datos, está primordialmente orientado por lo cualitativo, donde la forma en que los actores construyen el fenómeno estudiado, es el eje central de la investigación. La investigadora sustenta la naturaleza epistemológica y teórica, a través de un abordaje hermenéutico introduciendo el contenido y lo dinámico, integrando las experiencias en colectivo contextualizadas, diálogos desde una relación horizontal con procedimiento dialéctico y método descriptivo tomando como base la reflexión crítica, e interpretativa. 
Haciendo una dicotomía, entre lo manifestado en el desarrollo de la Declaración Mundial UNESCO (1998), artículo 9, denominado Métodos Educativos Innovadores: pensamiento crítico y creatividad, y la proposición inherente al trabajo de investigación en proceso, se considera muy relevante el hecho de la integración escuela-comunidad para el fortalecimiento de los saberes sociocomunitarios; puesto que conduce al descubrimiento de potencialidades humanas en el arte del saber comunitario, además, ayuda a desarrollar en la localidad una visión de futuro compartido entre personas, organizaciones e instituciones para mejorar la calidad de vida; movilizando a todos los integrantes autores y actores del proceso educativo hacia una transformación y emancipación colectiva.

En otro orden de ideas, se presentan las derivaciones teóricas que sustentan el desarrollo del mismo referente al paradigma crítico reflexivo que determina esta indagación y el propósito de la investigación dándole bases al objetivo grupal que se desarrolla con la gente y para la gente como finalidad de la investigación Acción Participativa (IAP), de igual manera se hace referencia a la teoría socio critica, de las situaciones y del aprendizaje social.

\section{Sustentación teórica}

En relación al tópico, el presente estudio cuenta con una significativa gama de postulados y teorías, específicamente en Venezuela, las directrices del sistema educativo, están expresadas en la Ley Orgánica de Educación 2009, siendo ésta un documento articulado con la Constitución de la República Bolivariana de Venezuela de 1999 y de forma más procedimental en el Diseño Curricular del Sistema Educativo Bolivariano (2007: 10) el cual propone "La transformación del individuo como sujeto social, basado en los valores de libertad, unidad e integración". De hecho, la Ley Orgánica de Educación (2009), plantea en su Artículo № 4. 
La educación como derecho humano y deber social fundamental orientada al desarrollo del potencial creativo de cada ser humano en condiciones históricamente determinadas, constituye el eje central en la creación, transmisión y reproducción de las diversas manifestaciones y valores culturales, invenciones, expresiones, representaciones y características propias para apreciar, asumir y transformar la realidad.

Actualmente, el Ministerio del Poder Popular para la Educación (MPPPE) mancomunadamente con organismos públicos, trabaja en fortalecimiento de la valoración de la identidad nacional mediante la Resolución 015 del Viceministerio de Comunidades Educativas y Unión con el Pueblo, el cual plantea un compromiso de trabajar por el desarrollo sociocultural de las comunidades y escuelas, en el marco del Movimiento por la Paz y la Vida. Haciendo referencia a la señalado, a su vez, activa los espacios escénicos de las instituciones educativas a través de la programación de una Grilla Cultural como parte del Plan de Masificación de las Artes y las Culturas para hacer de nuestras escuelas territorios de paz y libres de violencia, desde las representaciones más genuinas de hechos históricos, fiestas tradicionales y otras efemérides, con la participación activa y corresponsable de la misma comunidad en el ser y hacer de las regiones.

No obstante, en el escenario planteado la realidad venezolana en la actualidad experimenta carencias en valores, identidad, cultura, autoestima, entre otros, algo que contrapone la teoría expresa en el párrafo anterior. Puesto que la cultura como parte del reconocimiento, valoración y respeto a la identidad cultural de los pueblos implica múltiples interacciones entre creencias, tradiciones, bailes, danzas, historias, relatos que son elementos que conforman el tejido cultura y contribuyen al logro de la calidad educativa. En relación con esta idea, es importante señalar que el planteamiento viceministerial no se equipara con la cotidianidad y el sentir propio, sino que, los y las docentes encargados del cumplimento de esta tarea lo ven como 
lineamientos y trabajos lo hacen como un requisito administrativo o como acatamiento de directrices educativas.

En este sentido, en un proceso homogeneizador que tiene como fin los saberes comunitarios en la praxis educativa se podría señalar que se estaría abriendo un nuevo espacio a los valores culturales de los sectores venezolanos y especialmente al sector en estudio, cuyos soportes, estarían sustentados en la globalización y descentralización de la educación popular, pero que en la actualidad tiene su contra parte en las políticas de estado que son partidarios de una globalización humanizada, y en este sentido la UNESCO (2003), citada por Pérez, I. (2004), ratifica su apoyo al fortalecimiento del patrimonio de los pueblos, por considerarlo para ser utilizado en el hecho académico escolar - social como la más rica fuente de sabiduría de los pueblos y como identidad patrimonial.

\section{Sustentación Metodológica}

En esta investigación se trabajara con Investigación Acción Participativa, como un proceso cíclico de reflexión-acción-reflexión así lo platean Kemmis, S. y Mactaggart, R. (1998), tomando en cuenta la dialéctica que se establece en los actores sociales, es decir la interacción continua del proceso cíclico como visión pragmática del mundo social, en función de transformar la práctica educativa a través de la planificación estratégica en un contexto social rural fundamentado en el dialogo constante con la finalidad de consolidar el aprendizaje mutuo; con el énfasis en el diseño regenerativo continuo de sí mismo, con la metodología crítica dialéctica de transformación continúa de la praxis educativa y el fortalecimiento de los saberes sociocomunitarios. Así mismo, en algunas de estas reflexiones Elliott, J. (1990), coincide en que si mejora la práctica es porque alguien se esfuerza en que esto suceda, es decir cambian las acciones, las ideas, los contextos. 
Para lograr tal propósito, la investigación toma estos argumentos por ser el foco para la estrategia de enseñanza - aprendizaje, igualmente el investigador forma parte del equipo profesoral en busca de cambios de la realidad. De acuerdo a Park, P. (1992:141) "la investigación acción participativa se articula diferencialmente del método científico convencional, ya sea instrumental o interactivo". Bajo esta perspectiva la estructura metodológica traduce la planificación, diagnóstico, participación, triangulación, análisis, síntesis, reflexión, interacción e integración de los actores involucrados en el proceso investigativo.

\section{Ciclo de la Investigación y Resultados parciales}

El ciclo de la investigación que se desarrolló durante el Apartado I comprende varios procesos que se describen a continuación:

a). Aproximación a los informantes observando la realidad para generar reflexiones sobre la praxis educativa.

Siguiendo la estructura del modelo de pensamiento crítico, en relación a la recolección de datos y análisis de la información, se ha venido desarrollando tomando los lineamientos de diversos teóricos. De acuerdo a Arias, F. (2006: 53) "Las técnicas son las distintas formas o maneras de obtener información". En concordancia con el autor, las técnicas son un conjunto de estrategias que la investigadora aplica para recolectar la información requerida para su investigación, son las que sirven de recolección de datos para sistematizar la información recabada. En efecto, en la Acción Participativa para la investigación en proceso se utilizan entre otras técnicas: conversatorios en colectivo, entrevistas a profundidad y entrevistas grabadas, la observación participativa, diálogos reflexivos, la fotografía, libreta de notas, donde todos los autores inmersos aportan su respectiva experiencia.

En esta línea de pensamiento, la investigadora procede a realizar el análisis colectivo de la práctica tratando de indagar en las concepciones 


\section{subyacentes en ellas para confrontarlas con la realidad observada,}

documentación, categorización, contrastación y reflexión que orienta nuestra acción educativa, pues la confrontación de lo registrado con nuestras concepciones produce una contradicción que genera la transformación.

Tabla № 1. Sistematización de un Diálogo Reflexivo.

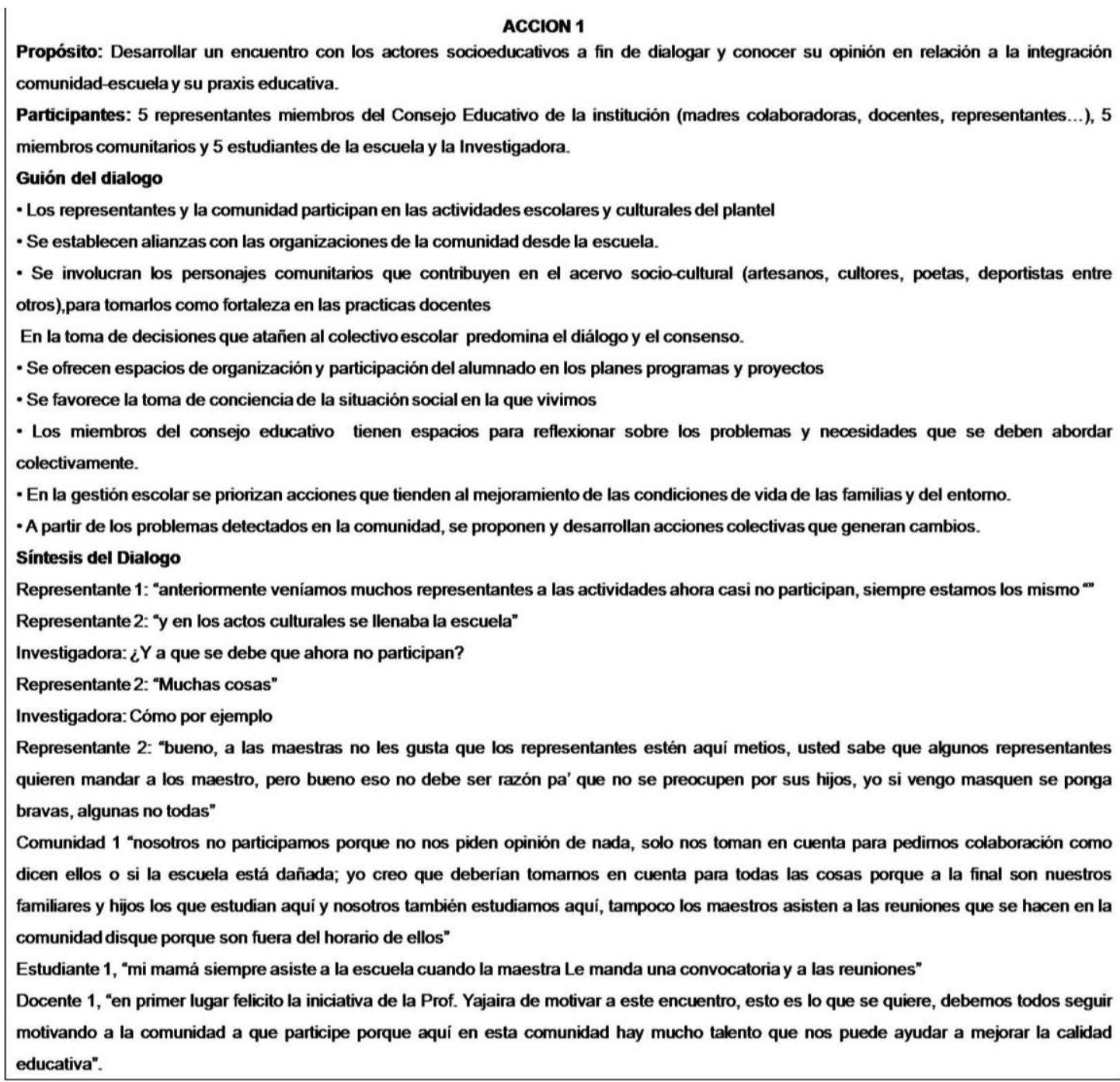

Fuente: Yajaira Araujo (2016). 


\title{
b). Accionando estrategias para abordar problemática de estudio
}

La transformación de la práctica sólo es posible con la participación activa de todos los involucrados en la evolución educativa para su mejora; por lo tanto, una vez que el colectivo identifica los problemas prioritarios, se definen las líneas de acción generales, se planifican y desarrollan las actividades que pueden generar cambios significativos en la realidad planteada. Para ello, se hace necesario desplegar procesos de integración y de discusión en colectivo, los cuales proporcionan herramientas teóricoprácticas a los métodos de enseñanza que brindan opciones concretas para nuestra acción en el contexto socioeducativo.

Tabla № 2. Conversatorio: propuestas a partir del análisis del contexto de la comunidad.

\begin{abstract}
ACCION 2
Propósito: Establecer propuestas de trabajo mancomunadas para transformar realidad educativa

Participantes: Comité académico, familia-comunidade investigadora.

-Comité Académico: “La escuela debe proyectar más su acción hacia la comunidad y no quedarse como una burbuja trabajando sólo con los programas que emana el Ministerio; para lograrlo, debemos comunicamos más con la comunidad y conocer cuáles son los temas que alli se necesitan más e integrarlos con las actividades que se hacen en la escuela aprovechando sus saberes que son muchos en lo artístico, deportivo, comercial_..." (Docente).

-Investigadora: “La realidad social es tan compleja y lenta de superar, que se hace necesario diseñar acciones tendientes a levantar la autoestima personal y social de la comunidad, con el propósito de despertar la identidad, la participación y los liderazgos. El aspecto cultural también reclama una atención urgente a través de la intervención de la escuela como ente rector de formación y preservación de los saberes de los pueblos, valorando los libros vivientes, tradiciones, artesanias entre otros aspectos importantes que caracterizan este hermoso terruño Merideño. Es imprescindible atender la formación sociopolitica de los líderes y brindarles apoyo en la construcción de los proyectos comunitarios. La escuela desde su ideario y peso ético, está llamada a fortalecer el tejido social del sector, como ente articulador de grandes iniciativas que apuntalen al desarrollo de la comunidad; siendo la investigación en proceso una de las respuesta más efectiva a tales propósitos".

-Familia-Comunidad "Yo diria que dos cosas desde mi punto de vista, uno: tenemos que partir de que la escuela no puede solucionar aislada de la comunidad problemas que se presenten, pero sí puede establecer esas relaciones con la gente, con los grupos organizados que pueda iluminar y promover un poco el trabajo de gestión comunitaria, por otro lado, de servirle de apoyo, para que el sector pueda mantener su cultura con miras a que se transmita de generación en generación. La otra parte más centrada hacia los alumnos, para que nuestros hijos vayan siendo más conscientes de la realidad en que viven y valoren lo que tienen en su comunidad" (Miembro de la Comunidad).

-Investigadora: "yo creo que por esa parte habria que enfocar un poco el trabajo, por supuesto en la medida en que se va trabajando con los alumnos debe vincularse a la familia, a los padres al entomo; para que el niño vaya valorando ya, dónde vive, cuál importante es el medio y personas que lo rodean, qué puede ir haciendo, para mantener sus raices. A la medida que los niños vayan siendo conscientes pues, irá surgiendo también, el amor a su cultura e idiosincrasia" (coordinadora de la escuela).
\end{abstract}

Fuente: Yajaira Araujo (2016). 
c). Extracto de la experiencia y la reflexión en y sobre la acción transformadora. Recogida la información, la investigadora procede a su organización para la sistematización de un relato inicial que le permitió ordenar y recuperar los datos obtenidos; para lo cual, se utilizó una libreta de campo, se recopilo evidencias físicas como: documentos, fotografías, grabaciones, registros de entrevista, entre otros. A partir de la relectura individual y colectiva de este relato inicial, es necesario superar la narración o descripción de la experiencia para generar interpretaciones donde se comparen el saber previo con el aprendido durante la práctica y se contraste éste último con los aportes de otros autores que nos ayuden a producir un nuevo conocimiento que brinde respuesta a la problemática detectada.

Tabla № 3. Extracto del relato de una vivencia.

\begin{abstract}
AGGION 3
En La Unidad Educativa Bolivariana Los Llanitos de Timotes, se ha venido revisando nuestro quehacer educativo encontrándose con resultados poco satisfactorios en relación con la apropiación de saberes comunitarios y la praxis educativa, que permitan aprendizajes vivenciales a los alumnos. Dichos resultados son similares a los hallados en los monitoreos pedagógicos dírigidos por la coordinación pedagógica del Municipio Escolar Miranda. Para dar respuesta a esta probłemática común, la investigadora pone en práctica el desarrollo del Proyecto de Tesis Doctoral cuyo propósito general es "La Integración de las Saberes Comunitarias como estrategia de aprendizaje en la praxis educativa de la Comunidad Escolar Los Lanitos de Timotes"

Para akanzar este propósito se hace necesario, en un primer momento tener un diagnóstico inicial de todos componentes de la acción eduçativa. Para ello la investigadora invita al colectivo socioeducativo a formar parte de una investigación para el año 2014 , en que se iniciaba con una revisión histórica-social en el contexto de la comunidad de estudio recabando información relevante de su trayectoria. Se recuerda aquella mañana en la que se dio el acercamiento institucional se solicitó al directivo y personal en general permiso respectivo para desarrollar el proyecto; todos los docentes callados se miraban, alguien dijo: me parece bien que revisemos y mejoremos nuestro trabajo educativo para hacer de nuestra escuela un centro de saberes comunitarios; aceptando en su totalidad la propuesta, se evidencio el entusiasmo con la idea.

El director, coordinadora pedagógica y los docentes del centro asumimos el reto de revisar qué estamos haciendo, reflexionar sobre nuestra práctica y descubrir qué estamos dejando de hacer, cuáles son nuestras debilidades y qué debemos mejorar en beneficio de la comunidad escolar, ya que los resultados obtenidos en relación a: identidad, arraigo cultural, el ser, el reflexionar y el convivir de nuestros alumnos no eran los mejores ni los esperados. Una vez asumido el compromiso nos reunimos en varias oportunidades, consejo educativo y comunidad, para conformar el colectivo coinvestigador quedando constituido por 4 docentes, 4 estudiantes, 1 madre colaboradora, la ambientalista, 4 representantes, 4 miembros de la comunidad y apoyo del equipo directivo del plantel que posteriormente tendremos la tarea de llevar a cabo los planes de acción transformadora y lograr el propósito planteado.

Formar parte de esta investigación significa un compromiso por parte de todo el colectivo coinvestigador ya que hemos tenido que revisar como docentes nuestra praxis educativa y como comunidad la importancia de la integración socioeducativa analizando las diferentes fuentes de información teórico-metodológicas, así como entrevistas, fotos, grabaciones, registros sistematizados y participar en las actividades requeridas para obtener información del proceso educativo. Entre las actividades realizadas para descubrir las realidades institucionales, hicimos las observaciones participantes, dialogo reflexivo de un día, registrando por escrito todo cuanto observamos. Para ello, nos dividimos el trabajo: unos observaron y realizaron registros cerrados en la libretas de notas, otros, registro fotográfico, la investigadora como moderadora del dialogo reflexivo.

Una vez analizada y sistematizada la información se dio una socialización grupal de los resultados del conversatorio entre ellos los más relevantes:

- La escuela debe proyectar estrategias hacia la comunidad aprovechando sus saberes e incorporarlos a la praxis educativa-

-Se hace necesario diseñar acciones tendientes a levantar la autoestima personal y social de la comunidad, con el propósito de despertar la identidad, la participación y los liderazgos.

-La escuela debe servir de apoyo pedagógico para que el sector pueda mantener su cultura con miras a que se transmita de generaciónen generación

-Vincular a la familia, a los padres al entorno al proceso educativo; para que los alumnos sean más conscientes de la realidad en que viven y valoren lo que los rodea.

Esta experiencia nos permitió reflexionar que en todos los procesos que se dan en la escuela estân implicitos dos vertientes o interrogantes: ¿que tenemos? y ¿hacia dónde vamos?, quedando esto como compromiso de trabajo colectivo.
\end{abstract}

Fuente: Yajaira Araujo (2016). 


\section{Categorías:}

\subsection{Cultura}

Con referencia a esta categoría Morín, E. (1999:26:), señala que "la cultura acumula en sí lo que se conserva, transmite, aprende; ella comporta normas y principios de adquisición". Visto de esta manera, la cultura implica una gama de saberes y haceres de las comunidades, la cual los identifica como pueblo. Por esta razón, las instituciones educativas necesitan valorar continuamente el quehacer cultural, las manifestaciones, costumbres y tradiciones de cada entidad, para evitar los embates de la aculturación y las consecuencias nocivas para futuras generaciones

Debe señalarse que, dentro de los hogares de la comunidad en estudio, se respira una paz, su gente se caracteriza por el respeto y la humildad, dentro de la misma se observan imágenes religiosas, también fotos para recordar acontecimientos importantes. Asimismo, a través de diálogos abiertos con libros vivientes se mostró el respeto y devoción por las festividades religiosas, cantautores locales, gastronomía en la cual se destaca la Chicha de la Sra. Flor, cultores, como el Sr. Williams apodado "El Poeta" entre otros. Este acervo cultural identifica el sector, el Municipio Miranda y la Región Andina Merideña, revalorizando y fortaleciendo la vida espiritual de su gente, motivo que conlleva a la reorientación y/o transformación de la praxis educativa a través del protagonismo consciente de sus actores.

\subsection{Tradición}

La tradición y la innovación en la cultura debe enfocarse desde una visión transformadora; no se trata de suplantar lo viejo por lo nuevo, ni de oponer la cultura tradicional a las formas y manifestaciones nuevas, como si fueran hechos contradictorios. Proceder así sería un gran error, por cuanto el antagonismo no se da entre sectores distintos de la cultura -ellos son perfectamente legítimos ya sean nuevos o tradicionales- sino que se presentan 
precisamente por la globalización, de allí el saber articular ambas tendencias sin perder lo autóctono ni la autonomía que las definen (Monsonyi, E., 1982).

Este señalamiento, permite reflexionar sobre la realidad actual donde la tradición no puede verse como algo ajeno o alejado de la cultura, ni tampoco como una rémora del pasado que nos impide ver el presente y asumir el futuro. El pasado representa nuestras raíces y que debe estar de la mano con orgullo, para ser traspasado de generación en generación, no bajo esquemas inamovibles que impidan nuestro progreso y adaptación a nuevas ideas, sino por el contrario nos permitan un piso sólido en el cual sus enseñanzas desarrollen en nosotros un espíritu crítico capaz de dar continuidad a nuestro pueblo, como lo expresa Mario Briceño Iragorry (1989).

En referencia a lo planteado, la investigadora realizó unas visitas por la comunidad en estudio, observando cómo se conservan algunas tradiciones con respecto a los días de descanso o guardar, como ellos le llaman coloquialmente, días santos, actos religiosos y costumbres propias, como el significado de las danzas a San Benito, La Virgen de Coromoto, San Isidro, así como la Paradura del Niño Jesús, cantos versos y villancicos entre otros.

\subsection{Identidad}

Otra categoría inherente a la investigación es la Identidad, que según Morín, E. (ob.cit.: 28), manifiesta que "las culturas están aparentemente encerradas en sí misma para salvaguardar su identidad". En atención a esta realidad, la identidad constituye el amor hacia lo nuestro, el respeto como base de toda convivencia humana y la preservación de las raíces culturales y por ende del patrimonio como legado a las generaciones futura.

Sin duda la riqueza cultural de los venezolanos, y en el caso de la Comunidad Los Llanitos, es evidente; eso que llamamos lo nuestro, y que es nuestro Patrimonio, sin embargo, al hablar de identidad debemos captar que no somos puros o únicos, producto de la transculturación que durante siglos 
hemos sufrido, sin embargo, debemos corregir la aculturación globalizada mediante la praxis educativa que fomente las raíces ancestrales y su perpetuidad en el tiempo.

\subsection{Saberes}

Para entrar en esta categoría implica tomar nuevamente las ideas de Morín, E. (ob.cit.: 28), donde expresa que "la cultura está constituida por el conjunto de los saberes, saber-hacer, reglas normas...creencias, ideas, valores, mitos que se transmiten de generación en generación”. En atención a lo expuesto, la sociedad encuentra en la triada escuela-familia-comunidad las principales fuentes de sabiduría. De esta forma, la escuela puede interrogarse a sí misma, a sus actores, a su contexto y al mismo tiempo aportar soluciones a los problemas; por lo tanto, se debe asumir la integración colectiva en la praxis educativa para la formación holística del individuo.

\subsection{Valores}

Por el solo hecho de que los valores son las cualidades del ser, sirven para evaluar nuestras propias acciones y las de los demás y sobre todo sirven para guiar el comportamiento de las personas y para identificar la cultura de un pueblo. Al respecto Buxarrais (1997: 81), citado por Torres, D. (2011: 33), considera que "Los valores tiene múltiples caras y pueden contemplarse desde variados ángulos. Los valores son importantes por sí mismos y dependen del momento histórico, cultural y la situación física donde surgen". Así pues, la conducta humana se halla condicionada y estimulada por las necesidades e intereses que tiene la persona no solo a nivel individual sino también colectivo, por lo que cada grupo social conforma un conjunto de normas, saberes, creencias y aspiraciones, valores que transmiten a sus miembros. 


\section{Conclusiones y reflexiones}

Profundizar la vida social, permite visualizar la realidad que vivimos y el dinamismo de transformación con niveles acelerados que nos dictan el proceder de las conductas actuales de los pueblos y como la aculturación ha creado modelos tergiversados del accionar ciudadano, presentado parámetro diferentes de conducta y comportamientos. Cabe destacar, que la transculturación, siempre ha tendido a colocar por encima la "cultura del otro", como la que de alguna forma nos conecta con el mundo externo, y la escuela como centro de formación debe no solo transmitir conocimientos pedagógicos sino además crear una transformación social, cultural en valores y saberes de identidad, integrando a la comunidad como fuente de sabiduría para enriquecer la praxis pedagógica a través de estrategias de aprendizajes dinámicas creando espacios abiertos donde se dé a conocer el patrimonio, los saberes ancestrales y el quehacer cultural.

En definitiva, a modo de reflexión los valores son muy importantes para la sociedad porque están presentes en todas las situaciones que vivimos a diario, en todas las decisiones que tomamos y se adquieren sobre todo a través de la práctica y del ejemplo; en ese diario convivir, compartir y expresan nuestro sentir en un momento determinado. Esta allí palpable y debe ser obligatorio que recuperemos la posibilidad de integrar a los actores sociales para la transmisión de saberes y de esta forma innovar la construcción curricular.

\section{Referencias}

Arias, F. (2006: 53). El Proyecto de Investigación. Guía para su elaboración. Editorial Epísteme. Orial Ediciones. 5ta. Edición. Caracas.

Briceño, M. (1989: 305-306). Doctrina Historiográfica. Obras Completas, Vol. 4. Ediciones del Congreso de la República. Caracas. 
Constitución de la República Bolivariana de Venezuela (1999). Gaceta Oficial № 36.860. Venezuela.

Declaración Mundial sobre Educación Superior en el siglo XXI UNESCO (1998). art. 9. Métodos Educativos Innovadores. Pensamiento Crítico y Creatividad.

Currículo Nacional Bolivariano (2007). Diseño curricular del sistema educativo Bolivariano. Caracas, Venezuela.

Elliot, J. (1990). La investigación acción en educación. Ediciones Morata, S.A. Madrid, España.

Kemmis, S. y Mactaggart, R. (1998). Cómo planificar la Investigación Acción. Editorial las Artes. Madrid.

Ley Orgánica de Educación (2009). Resolución Viceministerial 015. Gaceta Oficial № 5.929, del 15 de agosto. República Bolivariana de Venezuela: Viceministerio de Comunidades Educativas y Unión con el Pueblo.

Matos, Y. (2012). Participación activa de los actores sociales hacia una recreación sana. Trabajo de Grado presentado en la UNESR para optar al título de Magister en Educación.

Monsonyi, E. (1982). Identidad Nacional y Culturas Populares. Editorial La Enseñanza Viva (Serie: Identidad Nacional). Caracas.

Morín, E. (1999:26-28). “Los Siete Saberes Necesarios para la Educación del Futuro" Magisterio. Colombia.

Park, P. (1992:141). Qué es la Investigación Acción Participativa. Perspectivas teóricas y metodológicas. Editorial Popular. Colombia.

Pérez, I. (2004). "EI uso escolar-académico del habla Zuliana". Revista Venezolana de Ciencias Sociales. UNERMB. Vol. $8 \mathrm{~N}^{\circ} 1,111-125$ Cabimas, Venezuela. 


\section{Yajaira Esperanza Araujo Araujo}

e-mail: araujoyajairaunica@hotmail.com

Nacida en Timotes. Estado Mérida, Venezuela. Cursa

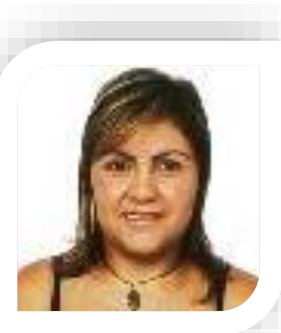

estudio de Doctorado en Ciencias de la Educación en

la Universidad Nacional Experimental Rafael María

Baralt (UNERMB) Estado Trujillo. Magister

Scientiarum en Administración de la Educación

Básica Universidad Nacional Experimental Rafael

María Baralt (UNERMB), Estado Trujillo. Licenciada en Educación Integral Universidad Cecilio Acosta, Valera Estado Trujillo. Técnico Superior Universitario en Educación Preescolar Instituto Universitario de Tecnología Mario Briseño Iragorri, Estado Trujillo. Técnico Medio Mención Administración de Personal Liceo Pedro García Leal, Estado Trujillo. Actualmente Coordinadora Pedagógica en el Núcleo Escolar Rural № 196 Estado Mérida desde el 2013.

El contenido de este manuscrito se difunde bajo una Licencia de Creative Commons ReconocimientoNoComercial-Compartirlgual 4.0 Internacional 